\title{
Elder women's perceptions around optimal perinatal health: a constructivist grounded-theory study with an Indigenous community in southern Ontario
}

\author{
Sujane Kandasamy MSc, Meredith Vanstone PhD, Mark Oremus PhD, Trista Hill, Gita Wahi MD MSc, \\ Julie Wilson BHSc (Midwifery), A. Darlene Davis RN, Ruby Jacobs BScN, Rebecca Anglin MD PhD, \\ Sonia Savitri Anand MD PhD
}

Video available at: http://cmajopen.ca/content/5/2/E411/suppl/DC2

\section{Abstract}

Background: Women play important roles in translating health knowledge, particularly around pregnancy and birth, in Indigenous societies. We investigated elder Indigenous women's perceptions around optimal perinatal health.

Methods: Using a methodological framework that integrated a constructivist grounded-theory approach with an Indigenous epistemology, we conducted and analyzed in-depth interviews and focus groups with women from the Six Nations community in southern Ontario who self-identified as grandmothers. Our purposive sampling strategy was guided by a Six Nations advisory group and included researcher participation in a variety of local gatherings as well as personalized invitations to specific women, either face-toface or via telephone.

Results: Three focus groups and 7 individual interviews were conducted with 18 grandmothers. The participants' experiences converged on 3 primary beliefs: pregnancy is a natural phase, pregnancy is a sacred period for the woman and the unborn child, and the requirements of immunity, security (trust), comfort, social development and parental responsibility are necessary for optimal postnatal health. Participants also identified 6 communal responsibilities necessary for families to raise healthy children: access to healthy and safe food, assurance of strong social support networks for mothers, access to resources for postnatal support, increased opportunities for children to participate in physical activity, more teachings around the impact of maternal behaviours during pregnancy and more teachings around spirituality/positive thinking. We also worked with the Six Nations community on several integrated knowledge-translation elements, including collaboration with an Indigenous artist to develop a digital story (short film).

Interpretation: Elder women are a trusted and knowledgeable group who are able to understand and incorporate multiple sources of knowledge and deliver it in culturally meaningful ways. Thus, tailoring public health programming to include elder women's voices may improve the impact and uptake of perinatal health information for Indigenous women.

\footnotetext{
Tn Canada, Indigenous peoples encompass 3 different groups: First Nations, Metis and Inuit. Within these groups, there are many cultural, linguistic and geographical differences. According to the National Household Survey, there are over 1.4 million Indigenous peoples in Canada, accounting for $4.3 \%$ of the total Canadian population. ${ }^{1}$ There was a $20.1 \%$ increase in these populations between 2006 and 2011 (compared to an increase of 5.9\% in the non-Indigenous population nationally). ${ }^{1}$

Indigenous peoples in Canada experience significant health disparities with regard to chronic health conditions such as cardiovascular diseases, arthritis, diabetes, asthma, bronchitis, kidney disease and cancer, ${ }^{2-5}$ with prevalence rates being
}

higher on reserve (especially for cardiovascular diseases and diabetes) than off reserve. ${ }^{6}$ In addition to health inequities, Indigenous peoples are challenged by a history that is intertwined with residential schooling and the "Sixties Scoop" (the taking of Indigenous children and placing them in foster

Competing interests: None declared.

This article has been peer reviewed.

Correspondence to: Sonia Anand, anands@mcmaster.ca

CMAJ Open 2017. DOI:10.9778/cmajo.20160077 
homes or adoption beginning in the 1960s and continuing until the late 1980s). ${ }^{7}$ These practices have led to the loss of language, culture and traditional ways of living, ${ }^{8}$ resulting in intergenerational loss and grief. 9

A chart review completed in collaboration with a First Nations community, the Six Nations of the Grand River, showed that prepregnancy body mass index, excess gestational weight gain and tobacco use during the gestational period are key areas of concern. ${ }^{10}$ These health disparities place Indigenous children at higher risk for chronic conditions, which highlights the importance for exploring avenues for improving maternal health. The decisions women make during pregnancy are affected by health beliefs and cultural perceptions, often shaped by the advice and support of experienced elder women. In Indigenous cultures, elder women play a double role as advisors and caregivers to subsequent generations. These 2 facets of elder women's roles lie in harmony, contributing to the intergenerational transmission of cultural norms and the assurance of optimal development. ${ }^{11-15}$

Pivoting on the cultural framework that elder Indigenous women play a key role in supporting and advising younger women during the perinatal period, we designed a qualitative study to investigate the perinatal health beliefs of elder Six Nations women. We also aimed to engage the community by developing culturally meaningful avenues for translating the knowledge co-created through this research.

\section{Methods}

\section{Context and setting}

Our research team has maintained a collaborative relationship with the Six Nations of the Grand River since 1999.1,10,16 Six Nations is the largest community of First Nations peoples in Canada. Six Nations comprises about 25650 Haudenosaunee people, 12271 of whom reside within the borders of the 18000 hectares of reserve land. ${ }^{17}$ Since Six Nations is a matrilineal society, women are at the head of the clan and play leading roles within the community, particularly around the transmission of culturally rooted health knowledge.

Although the Six Nations community is home to a diverse array of businesses, services and recreational programs, it also experiences socioeconomic hardships, including unemployment and low income, which are strongly associated with obesity, tobacco use, type 2 diabetes and cardiovascular disease. ${ }^{18}$ It is probable that socioeconomic hardships also interact with other risk factors such as nutrition, physical activity, mental health and domestic violence, thus contributing to adverse birth and newborn outcomes. ${ }^{19-21}$

\section{Design}

This is a qualitative study using a constructivist groundedtheory approach. ${ }^{22}$ The methods, including the adaptation of grounded theory to incorporate Indigenous knowledge, are described in detail elsewhere. ${ }^{16}$

In brief, we used an approach that incorporated diverse ways of doing, thinking and being, as informed and guided by a multidisciplinary team of Indigenous and non-Indigenous members. The project was established in concert with the Six Nations community through regular meetings, planning sessions and the development of a Six Nations advisory group. For example, the primary researcher (S.K.) worked with a community coder to ensure that the research outputs were resonant and helpful for those familiar with this research topic, to avoid imposing her own interpretations or subverting meaning, and to allow her to check her views in a reflexive way. The Six Nations advisory group provided leadership with project planning, participant recruitment, data collection, analysis and knowledge translation (including data dissemination).

We recruited Six Nations women who self-identified as grandmothers to participate in pilot-tested, semistructured individual or group interviews, as they preferred. Participants were not required to be biological grandmothers, as many older women from this community identified themselves as grandmothers if they fulfilled a caretaker role in their families or community. Our purposive sampling strategy was guided by the advisory group and included researcher participation in a variety of local gatherings to connect with people or groups who had contact with grandmothers within the community as well as personalized invitations to specific women, either faceto-face or via telephone. Participants also referred their friends/colleagues who met our eligibility criterion.

Interviews and focus groups lasted on average 60 minutes. They were conducted by a trained female graduate student (S.K.) and were audiorecorded and transcribed verbatim. Interviews took place in a private area at the participant's home or in a confidential room provided by the Six Nations Birthing Centre. At the time of the study, S.K. had many years of experience working with Indigenous communities and formal training in qualitative methodology. All interviews included an introductory period during which S.K. and the participant engaged in conversation that included, but was not limited to, self-introductions, discussions about the details of the study, personal interest in the research question, overall goals for the project, and obtaining informed written and oral consent for participation. The interview guide is given in Appendix 1 (available at www.cmajopen.ca/content/5/2/E411/suppl/DC1). Focus groups $(n=2)$ consisted of 3-7 women and were offered only on request. For example, the participants of the first focus group were all childhood friends who felt comfortable sharing personal information in front of one another.

We used the iterative process of data collection and analysis common to grounded-theory approaches. ${ }^{22}$ Analysis consisted of a staged coding process completed manually by 3 people: 2 qualitative researchers (S.K., G.W.) and a community member (T.H.). The 3 coders worked both independently and together to code and analyze all interviews. Data credibility was achieved through 4 clarifying and member-checking interviews with participants. Audio reflections, writing of postinterview memos and diagramming throughout the research process helped the primary researcher gain reflexive self-awareness of subjectivities, standpoint and avenues for increasing the level of deep listening during the interviews and focus groups. Data collection was stopped at the point of saturation, determined by group 
consensus as the point when new data were not leading to new information or new insights.

We also worked collaboratively with the Six Nations community on several integrated knowledge-translation and dissemination elements. In addition to forming an advisory group and working with a community coder, the primary researcher spent substantial time building relationships within the community, connecting with various organizations and participating in various cultural events and programs. Since the community had identified perinatal health as a priority area, there was much interest from people who wanted to be involved with this project in various capacities. We worked with an Indigenous artist to translate the study findings into a digital story (short film) and with the advisory group to distribute laylanguage summary reports during community gatherings.

\section{Ethics approval}

The study received research ethics approval from the Hamilton Integrated Ethics Board and the Six Nations Research Ethics Committee.

\section{Results}

All 18 women approached for this study agreed to be interviewed. Their demographic characteristics are given in Table 1. Three focus groups and 7 individual interviews were conducted. Four of the 18 women participated in supplementary member-checking interviews.

Primary beliefs about prenatal and postnatal periods The experiences of the participants converged on 3 primary

Table 1: Demographic characteristics of participants

\begin{tabular}{|lc|}
\hline Characteristic & $\begin{array}{c}\text { No. (\%) of } \\
\text { participants* } \\
(n=18)\end{array}$ \\
\hline Mean age (range), yr & $65(52-84)$ \\
\hline Median no. of grandchildren & 5 \\
\hline Marital status & $4(22)$ \\
\hline Married & $2(11)$ \\
\hline Common-law & $3(17)$ \\
\hline Single & $1(6)$ \\
\hline Divorced & $8(44)$ \\
\hline Widowed & $2(11)$ \\
\hline Highest level of education & $9(50)$ \\
\hline Less than high school & $1(6)$ \\
\hline High school & $4(22)$ \\
\hline Some college & $1(6)$ \\
\hline College diploma or equivalent & $1(6)$ \\
\hline Some university & \\
\hline Bachelor's degree & \\
\hline *Except where noted otherwise. & \\
\hline
\end{tabular}

beliefs about the prenatal and postnatal periods: 1) pregnancy is a natural phase of the life cycle, 2) pregnancy is a sacred period for both the woman and the unborn child and 3) immunity, security (trust), comfort, social development and parental responsibility are necessary for optimal postnatal health.

\section{Pregnancy is a natural phase of the life cycle}

Many participants perceived the state of pregnancy as a natural course of life that is not an illness, a medical problem (typically does not require medical intervention) or a condition that should be treated as a "comfort zone" or during which one should "baby" herself. When asked what advice she might give a pregnant woman, 1 participant said: "Don't sit for too long, don't be lazy, move around. You are not sick." (P4) Another participant stated:

And they can work and everything too, I think. You don't really need to baby yourself. Because to me, pregnancy is a normal process. Maybe they need to baby a little bit toward the end of the pregnancy when you need an afternoon nap or pillows to support your belly. (P7)

This understanding of pregnancy as a normal phase of life was identified through the participants' exhortations to avoid behaviours typically associated with illness, such as being sedentary for long periods ("lazing around"). Participants were clear in their belief that the state of pregnancy should not result in an intermission from normal, healthy habits (eating well, working hard and getting enough physical activity). Excess rest was considered by many to be unhealthy for the pregnant woman and for the growth pattern of her unborn child. Instead of lazing or laying around, one should continue rising with the sun and keeping up with one's prepregnancy chores and work life. For example, 1 participant said:

I don't think [the problem] is too much sleep, though. I think it's being lazy and not getting the exercise and moving around because if you are sitting or laying around doing nothing then your body is not going to be ready for the job of having a baby. It is a big job. (P6)

This practice of staying active and maintaining prepregnancy routines was thought to be important for getting the body ready for the job of childbirth, helping to ensure that the baby does not "settle" in a position that makes labour difficult, preventing excess weight gain, which can be difficult to shed, and avoiding the development of larger babies.

\section{Pregnancy is a sacred period for both woman and unborn baby}

Many participants described pregnancy as a symbol of a special spiritual state. It is a time when the pregnant woman and the unborn child are on the verge of 2 different spiritual worlds, and their fates are not easily guaranteed. When describing the uncertain nature of pregnancy, the participants explained that a woman should not be too prepared or too anxious for the baby's arrival.

They say that when you are getting ready for the baby to come, not to be too ready. Don't get cribs and all kids of toys and all 
kinds of gadgets and stuff like that because you don't know how long [the baby is] going to be here. And sometimes when you get too many things, [the baby isn't] going to stay long. Have the necessities but don't prepare too much. (P6)

Many participants also expressed that the sacredness of pregnancy necessitates that the pregnant woman stay vigilant, monitor all symptoms that occur in excess, be aware of changes that may occur, listen to her body and learn from her experiences. Learning from experience is how one learns how to trust oneself but also gains an understanding that the things that are out of one's control will be taken care of by a greater power.

Given the sacredness of pregnancy, the maintenance of balance is critical to the pregnant woman's well-being spiritual, physical, social and emotional. The participants expressed that if all these factors are balanced, the woman would not be faced with sadness, feeling down or feeling too excited about the baby's arrival. This concept extends to doing everything in moderation. For example, 1 grandmother stated "Everything in moderation is the goal. Between extremes. If you go too much the other way too, that's not good for your baby, to develop a normal, healthy baby." (P12) Another grandmother said "To me it's more of a spiritual thing. You need balance all around - physical, spiritual and mental, it all has to be balanced. And I just took time to myself to pray and sort through things and I was fine." (P7)

\section{Requirements for optimal postnatal health}

In discussions of postnatal health, the advice of many of the participants encompassed a mixed view of traditional understandings and modern medical knowledge. They described the postnatal period as a time that is marked by the building of adequate immunity, security (trust), comfort, social development and parental responsibility (Table 2).

\section{Community-level factors}

The participants identified 6 primary areas of concern for the development of healthy future generations: access to healthy and safe food, assurance of strong social support networks for mothers, access to resources for postnatal support, increased opportunities for children to participate in physical activity, more teachings around the impact of maternal behaviours during pregnancy and more teachings around spirituality/ positive thinking. These areas of concern are communal responsibilities that are believed to set a strong foundation for healthy Six Nations families. Many participants discussed the importance of healthy food at length, touching on concern over the following factors:

- Contaminants that enter the food chain as a result of industrial processes; the manipulation of food that once grew natively on the land (genetic modification)

- New childhood allergies as a result of prenatal food choices

- Lack of knowledge around familial medical histories (which can affect food choices, especially during pregnancy)

- Feeding sugary carbonated beverages to young children

- Lack of reverence toward traditional teas.

\section{Knowledge translation and dissemination}

The digital story (4-minute film) contains a spoken word piece (poem) composed by a Six Nations woman. The primary researcher worked with the artist to ensure the findings were translated accurately and with integrity. She also worked with a filmmaker to visually showcase the artist performing the poem and to select visual clips that reflect the symbolism within the poem. We made the film available for public view on various social media outlets including Facebook, Twitter and YouTube (https://goo.gl/rCvOYb and https://goo.gl/ $\mathrm{J} 1 \mathrm{aYiE})$. It has been viewed by thousands of people around the world and has been celebrated by the Six Nations community and Western scientists. ${ }^{23}$

\section{Interpretation}

This study explored the beliefs around perinatal health of elder Six Nations women. In general, their perceptions were focused around 3 primary beliefs about the prenatal and postnatal periods: 1) pregnancy is a natural phase of the life cycle, 2) pregnancy is a sacred period for both the woman and the unborn child and 3) immunity, security (trust), comfort, social development and parental responsibility are necessary for optimal postnatal health. The advice that they delivered also represents a combination of traditional and modern medical knowledge. For example, many participants stressed the importance of adhering to standard immunization protocols (which is common Western biomedical advice) and also of keeping babies at home for the first 3 months so that their immunity can develop properly (which is more traditional, cultural knowledge). The wealth of knowledge described through stories and advice of these Six Nations women represents a fruitful opportunity to include grandmothers as key support people in the care of pregnant women and families with young children. Elder women are a trusted and knowledgeable group who are able to understand, appreciate and incorporate multiple sources of knowledge and deliver this knowledge in culturally meaningful ways. Within an Indigenous context, tailoring public health programming and interventions to include elder women's voices may improve the impact and uptake of health information during the perinatal stages. Perhaps this can be achieved through perinatal health programs that are facilitated by elder women or featuring their stories (and advice) as the foundation of health messages. In addition, programs that connect women to local elders during perinatal health visits or for support during the postpartum period (e.g., breast-feeding support) can also be beneficial.

For many Indigenous peoples, birth is considered a communal event that is celebrated and believed to strengthen relationships between families and the local natural environment. ${ }^{24-26}$ This view is reflected in the participants' belief that there are many community-level responsibilities involved in ensuring optimal health for pregnant women, young children and families. Maternal nutrition was traditionally considered a community responsibility. However, the disruption of traditional lifestyles and diets brought on by the legacy of colonization and associated environmental degradation has been linked to a growing epidemic of obesity among Indigenous 
peoples. ${ }^{27,28}$ Participants also expressed concerns around access to and contamination and genetic manipulation of food. To address these concerns, it is important to use a community-based approach that benefits from multiple knowledge sources, including experts such as elders.
Many participants also identified postnatal care and support to be very important, particularly in regard to postpartum weight loss. In parallel, another study captured a perspective of Indigenous women, who described postpartum weight loss as "important but hard," thus identifying a need for

Table 2: Participants' understanding of requirements for optimal postnatal health

Requirement

Immunity

\section{Security is developed primarily through wrapping, bundling} and "snuggling" the infant and supporting predictable routines. Participants stated that when a child feels comfortable and secure, this helps to form stronger mother-child bonds, contributing to the optimal development of the child's mind (by helping him or her learn how to stay calm).

Comfort Comfort consists of ensuring that the baby is comfortable in her or his clothing, comforting the baby when he or she needs to be reassured, and how the baby is supported during sustained periods of sitting (e.g., use of a traditional Indigenous baby carrier [cradleboard] instead of a baby seat). Participants believed that feeling comfortable helps the baby to develop trust, confidence and the ability to calm her- or himself.

Immunity involves making a mindful effort to keep the newborn immunization protocols. This is a crucial element in the expressed the need to give the newborn adequate tim
develop her or his immunological defences within the boundary of the home environment before exposing the infant to new surroundings.

Illustrative quote

"We were supposed to stay home for the first 3 months, not visiting other homes or out in public. And mothers don't follow that anymore. If anybody wanted to see the baby, they would come here. There is a double duty of keeping the mother well and the baby not exposed to germs until she has built immunity. [Nowadays] right out of the hospital, they've got to go shopping, and do this and do that. They should take more time - to give [the baby] a chance to be healthy." (P4)

"Babies need to feel secure. That's what swaddling does for them. It helps them feel snuggled because they are not used to all this space. When they were inside mom they were all bundled up, and if they are still like that outside, babies are more calm." (P6)

"I dislike those baby seats. They would be a lot better if they were on a cradleboard. It's better for their bones and spine. It must be uncomfortable for [the baby]. They all protest, they all cry. That's definitely important. Making sure that baby is comfortable and not just buying things because they are available [and convenient]." (P3)

"It just bugs me when people take their babies out, I mean their eyes are new. They should keep them in a darker room for two weeks. But now you see women running around with little stiff clothes on [the babies] and stiff hats on their little heads. And jeans. Hard jeans on their soft little body. That just bugs the heck out of me." (P5)

Social Social development consists of ensuring reciprocal development communication (making eye contact and talking to the newborn) to help promote social growth and development. Participants believed that social development through active communication aids in teaching important skills that will help the newborn learn how to form satisfying and trusting relationships.

Parental responsibility
Parental responsibility includes being accountable for the child that one has brought into this world. It includes being available for the child and helping her or him become the productive person he or she was were meant to be. Participants recognized that returning to work postnatally is a personal choice for each individual woman. Many did encourage staying home with the baby instead of having someone else watch her or him, especially during the first year, which was felt was a crucial period for building a strong bond. Participants consistently stated that a woman should take responsibility for her children. Parental responsibility also encompasses ensuring that the woman is ready to raise children before conceiving, that she can commit time for them once they arrive, and that she continues to be there for them during challenging times such as parental separations.
"[The baby] knows who you are and all. He sees you, hears your voice, that's one thing that I told my grandchildren, 'Talk to your baby.' If you are going to change him, tell him that. He knows the tone of your voice, and if you keep talking to him, he's going to respond. Eventually. So that's the social development at a young age. Because they are born with that wonderful thing called a brain and they start to think right away. You might not know it, but they do." (P8)

"She should stay with the baby instead of having someone else watch [her]. If it was up to me to tell my daughter to stay home with her kids, I would tell her to stay home as long as she could with them." (P9) "There's quite a bit of abuse separation, you know. A lot of men leave their wives, and it could be the other way around, too, with leaving their husbands with the kids and taking off. You see a lot of that. We were always there for them. You have to be there for your children." (P10) 
improved culturally relevant, community-based support and services. ${ }^{29}$ These services could include more options for child care, improved transportation on reserves and providing access to health care providers who can offer culturally safe support to women during the perinatal period. Because these concerns are vital to ensuring a healthy life for subsequent generations, it is important to work closely with various community experts and policy-makers to develop a sustainable and timely plan to address these issues.

\section{Strengths and limitations}

Our rigorous methodology and integrated knowledgetranslation efforts contributed to enhanced community-level uptake of the findings. However, we studied a single Indigenous community in Canada, and therefore the results may not be generalizable to all Indigenous communities. As each community has its own unique practices, it may be difficult to apply the experiences of 1 reserve to other reserves.

\section{Conclusion}

This study contributes to the limited literature regarding elder Indigenous women's perceptions around optimal perinatal health. Elder women are a trusted and knowledgeable group who are able to understand and incorporate multiple sources of knowledge and deliver this knowledge in culturally meaningful ways. Thus, tailoring public health programming to include elder women's voices may improve the impact and uptake of perinatal health information for Indigenous women.

\section{References}

1. Aboriginal peoples in Canada: First Nations people, Métis, and Inuit. Ottawa: Statistics Canada; 2011. Available: https://www12.statcan.gc.ca/nhs -enm/2011/as-sa/99-011-x/99-011-x2011001-eng.cfm (accessed 2016 Mar. 1).

2. Anand SS, Yusuf S, Jacobs R, et al. Risk factors, atherosclerosis, and cardiovascular disease among Aboriginal people in Canada: the Study of Health Assessment and Risk Evaluation in Aboriginal Peoples (SHARE-AP). Lancet 2001;358:1147-53

3. Shah BR, Hux JE, Zinman B. Increasing rates of ischemic heart disease in the native population of Ontario, Canada. Arch Intern Med 2000;160:1862-6.

4. Yusuf S, Reddy S, Ounpuu S, et al. Global burden of cardiovascular diseases: part II: variations in cardiovascular disease by specific ethnic groups and geographic regions and prevention strategies. Circulation 2001;104:2855-64.

5. Sin DD, Wells H, Svenson LW, et al. Asthma and COPD among aboriginals in Alberta, Canada. Chest 2002;121:1841-6.

6. Loppie Reading C, Wien F. Health inequalities and the social determinants of Aboriginal peoples' bealth. Prince George (BC): National Collaborating Centre for Aboriginal Health; 2009. Available: www.nccah-ccnsa.ca/docs/social\%20determinates/ NCCAH-Loppie-Wien_Report.pdf (accessed 2015 July 1).

7. Wesley-Esquimaux C, Smolewski M. Historic trauma and Aboriginal healing. Ottawa: Aboriginal Healing Foundation Research; 2004. Available: www.ahf. ca/downloads/historic-trauma.pdf (accessed 2015 July 1).

8. McIvor O, Napoleon A. Language and culture as protective factors for at-risk communities. Int 7 Indig Health 2009;5:6-25.

9. Cull R. Aboriginal mothering under the state's gaze. In: Lavell-Harvard D, Lavell J, editors. Until our hearts are on the ground: Aboriginal mothering, oppression, resistance and rebirth. 1st ed. Toronto: Demeter Press; 2006:141-56.

10. Oliveira AP, Kalra S, Wahi G, et al. Maternal and newborn health profile in a First Nations community in Canada. 7 Obstet Gynaecol Can 2013:35:905-13.

11. Jernigan HL, Jernigan MB. Aging in Chinese society: a bolistic approach to the experience of aging in Taiwan and Singapore. New York: Haworth Pastoral Press; 1992.

12. Gryboski KL. Maternal and non-maternal time-allocation to infant care, and care during infant illness in rural Java, Indonesia. Soc Sci Med 1996;43:209-19.

13. Bender DE, McCann MF. The influence of maternal intergenerational education on health behaviors of women in peri-urban Bolivia. Soc Sci Med 2000; 50:1189-96.
14. Aubel J, Brasha E, Bejtja G, et al. The Grandmother Study: devotion and caring. Tirana (Albania): Child Survival Project, Albanian Red Cross; 2004.

15. Piperata BA. Forty days and forty nights: a biocultural perspective on postpartum practices in the Amazon. Soc Sci Med 2008;67:1094-103.

16. Kandasamy S, Vanstone M, Oremus M, et al. A case study of a methodological approach to co-creating optimal perinatal health knowledge between Western and Indigenous communities. Int 7 Qual Methods 2017;16:1-11.

17. Community profile. Ohsweken (ON): Six Nations of the Grand River; 2013 Available: www.sixnations.ca/CommunityProfile.htm (accessed 2016 June 20).

18. Anand SS, Razak F, Davis AD, et al. Social disadvantage and cardiovascular disease: development of an index and analysis of age, sex, and ethnicity effects. Int 7 Epidemiol 2006;35:1239-45.

19. Kramer MS, Séguin L, Lydon J, et al. Socio-economic disparities in pregnancy outcome: Why do the poor fare so poorly? Paediatr Perinat Epidemiol 2000; 14:194-210.

20. Séguin L, Potvin L, St-Denis M, et al. Depressive symptoms in the late postpartum among low socioeconomic status women. Birth 1999;26:157-63.

21. Bohn DK, Tebben JG, Campbell JC. Influences of income, education, age, and ethnicity on physical abuse before and during pregnancy. 7 Obstet Gynecol Neonatal Nurs 2004;33:561-71.

22. Charmaz K. Constructing grounded theory. 2nd ed. London (UK): Sage; 2014.

23. Congratulations to the 2015 IHDCYH Talks Video Competition winners! Ottawa: Canadian Institutes of Health Research; 2015. Available: www. cihrirsc. gc.ca/e/49617.html (accessed 2016 Jan. 22)

24. Benoit C, Carroll D, Chaudhry M. In search of a healing place: Aboriginal women in Vancouver's downtown eastside. Soc Sci Med 2003;56:821-33.

25. Celebrating birth - Aboriginal midwifery in Canada. Ottawa: National Aboriginal Health Organization; 2008. Available: www.naho.ca/documents/naho/ english/midwifery/celebratingBirth/Midwiferypaper_English.pdf (accessed 2015 Apr. 1).

26. Kornelsen J, Kotaska A, Waterfall P, et al. The geography of belonging: the experience of birthing at home for First Nations women. Health Place 2010; 16:638-45.

27. Young TK, Reading J, Elias B. Type 2 diabetes mellitus in Canada's First Nations: status of an epidemic in progress [published erratum in CMA72000; 163:1132]. CMA7 2000;163:561-6.

28. Lix LM, Bruce S, Sarkar J, et al. Risk factors and chronic conditions among Aboriginal and non-Aboriginal populations. Health Rep 2009;20:21-9.

29. Vallianatos H, Brennand EA, Raine K, et al. Beliefs and practices of First Nation women about weight gain during pregnancy and lactation: implications for women's health. Can F Nurs Res 2006;38:102-19.

Affiliations: Department of Health Research Methods, Evidence, and Impact (Kandasamy, Oremus, Wahi, Anand), McMaster University; Department of Family Medicine (Vanstone), McMaster University; Centre for Health Economics and Policy Analysis (Vanstone), McMaster University, Hamilton, Ont.; School of Public Health and Health Systems (Oremus), University of Waterloo, Waterloo, Ont.; Birthing Centre (Hill, Wilson), Six Nations Health Services, Ohsweken, Ont.; Department of Pediatrics (Wahi), McMaster University, Hamilton, Ont.; Six Nations Health Services (Davis); Six Nations Health Foundation (Jacobs), Ohsweken, Ont.; Departments of Medicine (Anglin, Anand) and Psychiatry and Behavioural Neurosciences (Anglin), McMaster University, Hamilton, Ont.

Contributors: Sujane Kandasamy and Sonia Anand conceived the study, and Sujane Kandasamy, Meredith Vanstone, Mark Oremus, Julie Wilson, Rebecca Anglin and Sonia Anand designed it. Sujane Kandasamy, Trista Hill, Darlene Davis and Ruby Jacobs helped recruit eligible participants. Sujane Kandasamy and Trista Hill acquired the data. Sujane Kandasamy, Trista Hill and Gita Wahi analyzed and interpreted the data. Sujane Kandasamy, Meredith Vanstone, Mark Oremus and Sonia Anand contributed to drafting the manuscript. Sujane Kandasamy, Meredith Vanstone, Mark Oremus, Gita Wahi, Darlene Davis, Ruby Jacobs, Rebecca Anglin and Sonia Anand revised the manuscript for important intellectual content. All of the authors approved the final version to be published and agreed to act as guarantors of the work.

Acknowledgements: The authors thank Tahnee Wilson and Bhavna Samtani for their creative expertise in designing and executing the digital story (short film) as part of our integrated knowledge-translation efforts.

Supplemental information: For reviewer comments and the original submission of this manuscript, please see www.cmajopen.ca/content/5/27 E411/suppl/DC1 